\title{
Secondary prevention with folic acid: results of the Goes extension study
}

\author{
A Liem, G H Reynierse-Buitenwerf, A H Zwinderman, J W Jukema, D J van Veldhuisen
}

$\mathrm{H}$ omocysteine has been regarded as a modest independent risk factor for atherosclerotic vascular disease. ${ }^{12}$ Although folic acid can reduce homocysteine concentrations substantially, the value of folic acid intervention in primary and secondary prevention in terms of hard clinical end points is still uncertain. Many studies addressing this question are still underway. The results on folic acid intervention in patients undergoing percutaneous coronary intervention are not consistent. ${ }^{3}$ Recently we have reported the results of an open label intervention study with folic acid in a population with stable coronary artery disease while taking stable statin treatment. ${ }^{4}$ Within a follow up of 24 (10) months folic acid did not appear to attenuate the risk for recurrent events. Yet longer follow up observations are necessary before definite conclusions can be made. We therefore continued the study after renewed informed consent of the participating patients. We report here the results of the study with a mean (SD) follow up of 42 (10) months. In addition, we analysed the data again according to contemporary definitions of acute coronary syndromes (ACS). ${ }^{5}$

\section{PATIENTS AND METHODS}

The methods of the study have been previously reported. ${ }^{4}$ Briefly, consecutive patients with stable coronary artery disease visiting the outpatient department of the cardiology department were screened for inclusion. The history of patients had to include one of the following: myocardial infarction, significant coronary artery lesions $(>60 \%)$ on coronary angiography, percutaneous coronary intervention, or coronary bypass surgery. Patients had to be stable with no invasive vascular procedures scheduled. Patients were eligible when they had been taking statin for at least three months. The main exclusion criteria were age below 18 years, history of low vitamin B12 concentration, treatment for hyperhomocysteinaemia, severe renal failure or any other treatment for renal disease, known hepatic disease, signs and symptoms of severe heart failure (New York Heart Association functional class IV), or any other serious illness that would exclude the patient from follow up of at least three years.

The patients were randomly assigned to receive open label folic acid $0.5 \mathrm{mg}$ once daily or to standard care. In addition, during the study statin treatment was intensified when necessary. At least one of four goals was meticulously pursued: firstly, a decrement of low density lipoprotein (LDL) cholesterol of $30 \%$ (compared with concentrations before the initiation of statin treatment); secondly, LDL cholesterol concentration of $<3 \mathrm{mmol} / \mathrm{l}$; thirdly apolipoprotein B concentration of $<1 \mathrm{~g} / \mathrm{l}$; and fourthly, a decrement of apolipoprotein B concentrations of 30\% compared with prestatin concentrations. Persistent nicotine use was discouraged at regular intervals. Patients were followed up for a maximum of five years. During the whole study clinical events were carefully registered. Visits for laboratory examinations were planned at three, six, and 12 months and every six months thereafter. The study was conducted in

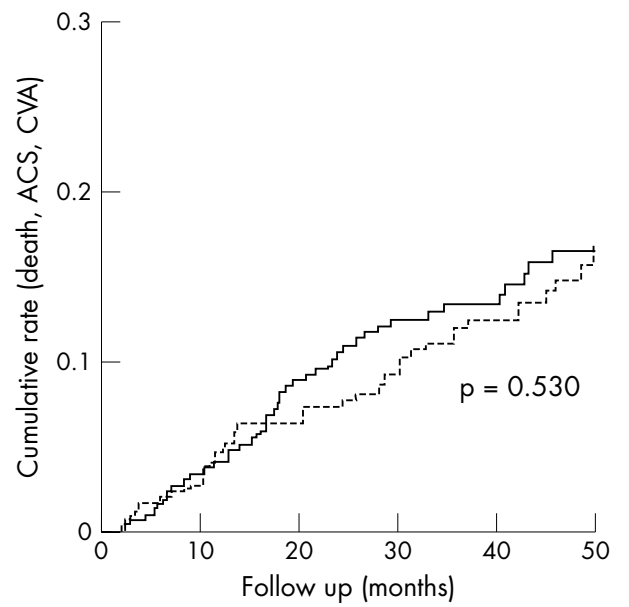

Figure 1 Cumulative event rate according to random allocation to folic acid treatment (dashed line) and control (solid line). Analysis for cumulative event rate for death, acute coronary syndromes (ACS) or cerebrovascular accident (CVA) or transient ischaemic attack. Probability value is estimated by log rank statistic.

accordance with the Declaration of Helsinki as revised in 1996. This study was performed in a rural area in the vicinity of the city of Goes, called the Bevelanden (province of Zeeland, the Netherlands), from 1998 to 2003. The local ethics committee approved the study protocol before the start of the study. The primary end point was a composite of vascular events. These events were defined as vascular death (sudden death, fatal recurrent ACS, fatal stroke, and other cardiovascular deaths), non-cardiovascular death, recurrent ACS, cerebrovascular accident, and transient ischaemic attack. ACS was defined according to contemporary criteria, which include an increase of troponin of $>0.2 \mu \mathrm{g} / \mathrm{l}$ (with a typical rise and fall) with at least one of the following: ischaemic symptoms, development of pathological Q waves on the ECG, and ECG changes indicative of ischaemia. All clinical events were adjudicated by an independent end point monitoring committee, unaware of treatment arm.

\section{RESULTS}

A total of 593 patients were enrolled in this study. Of these patients, 300 were randomly assigned to folic acid $0.5 \mathrm{mg}$ once daily and 293 patients served as control group. Baseline data have been previously reported. Mean follow up was 42 (10) months. After inclusion, 24 patients withdrew from the study (12 in each group) but were followed up according to the protocol and were included in the analysis on an intention to treat basis. No patients were lost to follow up. During the course of the study no difference in LDL concentrations between the groups were noted; on average LDL concentrations were $2.6(0.6) \mathrm{mmol} / \mathrm{l}$.

Clinical cardiovascular events were evenly distributed in both treatment arms. In total, 49 (16.3\%) clinical events in 
the folic acid group and 56 (19.1\%) in the control group were observed. In the folic acid group, 18 patients died of any cause (12 of vascular causes) and in the control group 26 patients died (17 of vascular causes). In the folic acid and control groups, 23 and 18, respectively, had ACS and eight and 12, respectively, had a cerebrovascular accident or transient ischaemic attack. The Kaplan-Meier curve in fig 1 depicts the time to the first clinical event. There were no significant differences between the groups (log rank test, $\mathrm{p}=0.53)$; the relative risk for total events was $0.85(95 \%$ confidence interval 0.56 to 1.31 ). This was also found for patients in the highest quartile of plasma homocysteine of $>13.7 \mu \mathrm{mol} /(\log$ rank test, $\mathrm{p}=0.31)$; the relative risk in this group was 1.37 (95\% confidence interval 0.65 to 2.87 ). Since no effect of folic acid intervention was observed, the study group as a whole was further analysed. As in the initial study creatinine clearance was the most pronounced laboratory risk factor. In a multivariate model corrected for sex, prior myocardial infarction, percutaneous coronary intervention, and coronary bypass surgery, homocysteine concentration disappeared as a significant risk factor when the creatinine clearance rate was introduced.

\section{DISCUSSION}

Our previous study had two limitations: the relatively short observation period of two years and the use of the old definitions of myocardial infarction. We therefore extended our observation period to almost four years and implemented the renewed definitions of myocardial infarction, which included increased troponin concentrations. Nevertheless, we obtained similar results, showing no benefit of folic acid intervention. Therefore, homocysteine has to be interpreted as a modifiable risk marker without apparent clinically salutary effects on hard clinical outcomes in patients with stable coronary artery disease until more evidence from other clinical trials becomes available.

\section{Authors' affiliations}

A Liem, G H Reynierse Buitenwerf, Department of Cardiology, Oosterscheldeziekenhuizen, Goes, the Netherlands

A H Zwinderman, Academic Medical Centre, University of Amsterdam, Amsterdam, the Netherlands

J W Jukema, Leiden University Medical Centre, Leiden, the Netherlands D J van Veldhuisen, University Hospital, Groningen, the Netherlands

Correspondence to: Dr A Liem, Department of Cardiology,

Oosterscheldeziekenhuizen, Post-box 106, 4460BB Goes, Netherlands; anho@zeelandnet.nl

Accepted 29 October 2004

\section{REFERENCES}

1 Wald DS, Law M, Mooris JK. Homocysteine and cardiovascular disease: evidence on causality from a meta-analysis. BMJ 2002;325:1202-9.

2 The Homocysteine Studies Collaboration. Homocysteine and risk of ischemic heart disease and stroke: a meta-analysis. JAMA 2002;288:2015-22.

3 Lange $\mathrm{H}$, Suryapranata $\mathrm{H}$, De Luca, et al. Folate therapy and in-stent restenosis after coronary stenting. N Engl J Med 2004;350:2673-81.

4 Liem A, Reynierse-Buitenwerf GH, Zwinderman AH, et al. Secondary prevention with folic acid: effects on clinical outcomes. J Am Coll Cardiol 2003:41:2105-13.

5 Trevelyan J, Needham EW, Smith SC. Sources of diagnostic inaccuracy of conventional versus new diagnostic criteria for myocardial infarction in an unselected UK population with suspected cardiac pain, and investigation of independent prognostic variables. Heart 2003;89:1406-10.

\section{IMAGES IN CARDIOLOGY}

\section{Cholesterol embolisation syndrome}

\begin{abstract}
A 68 year old male smoker, who was hypertensive, diabetic, and dyslipidaemic, with renal dysfunction (blood urea nitrogen $19 \mathrm{mg} / \mathrm{dl}$, serum creatinine $1.6 \mathrm{mg} / \mathrm{dl}$ ) and recent troponin $\mathrm{T}$ negative unstable angina, underwent a coronary angiogram. A non-ionic contrast agent (iopamidol) was used. Pre-procedure peripheral pulses were normal with no bruit. The angiogram revealed three vessel disease, and coronary artery bypass graft surgery was advised. Immediately following the angiogram no significant deterioration of renal function was evident. However, the patient presented four weeks later with renal failure (maximum blood urea nitrogen $73 \mathrm{mg} / \mathrm{dl}$, serum creatinine $3.6 \mathrm{mg} / \mathrm{dl}$ ), livedo reticularis (panel A, arrow), and bilateral persistent lower limb pain with bluish discolouration of toes suggestive of digital gangrene (panels A and B). Peripheral pulses were normal. Doppler of lower limb arteries showed normal flow in major arteries. A diagnosis of cholesterol embolisation syndrome (livedo reticularis and digital gangrene with renal impairment) was made. The patient was managed conservatively.
\end{abstract}

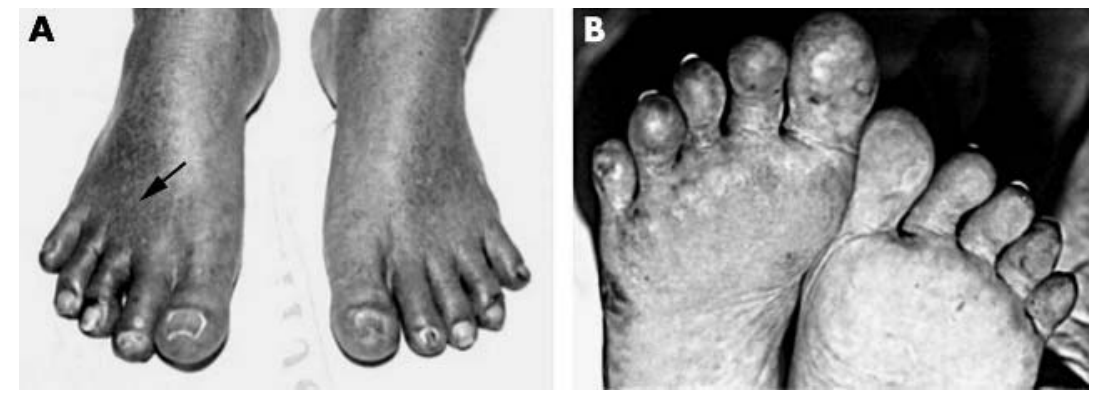

Cholesterol embolisation syndrome is a systemic disease caused by distal showering of cholesterol crystals after angiography, major vessel surgery, or thrombolysis. The management is supportive. The syndrome may go undetected unless there is monitoring of renal function following invasive procedures. 